\title{
The Impact of Enhanced Projector Display on the Responses of People to a Violent Scenario in Immersive Virtual Reality
}

\author{
Aitor Rovira \\ University College London \\ University of Barcelona
}

\author{
David Swapp \\ University College London
}

\author{
Richard Southern \\ Bournemouth University
}

\author{
Jian J. Zhang \\ Bournemouth University
}

\author{
Mel Slater* \\ ICREA - University of Barcelona \\ University College London
}

\begin{abstract}
This paper describes the impact of display resolution and luminance on the responses of participants in a behavioral study that used a projection-based Immersive Virtual Reality System. The scenario was a virtual bar where participants witnessed a violent attack of one person on another due to an argument about support for a soccer club. The major response variable was the number of interventions made by participants. The study was between-groups with 10 participants in two groups pre-upgrade and post-upgrade, both in the same 4-screen Cave-like system. However, the post-upgrade group experienced the scenario with projectors that had a significantly higher level of resolution and luminance than those experienced by the pre-upgrade group. The results show that, other things being equal, the number of both verbal and physical interventions was greater amongst those in the post-upgrade group compared to the pre-upgrade group.
\end{abstract}

Keywords: User studies, large format displays, Cave system, virtual characters, presence, bystander behavior.

Index Terms: I.3.7 [Computer Graphics]: Three-Dimensional Graphics and Realism-Virtual reality

\section{INTRODUCTION}

Successful applications of virtual reality in the psychological and behavioral sciences rely on people acting and responding realistically to virtual events and situations. This has been referred to as the sensation of presence [12] and a number of studies have sought to unravel various factors that may contribute towards such realistic response. Some technological factors studied have been the system used [11], the field of view [7], the frame rate [2], the use of a head tracking [3], the number of displays [4] and the latency between the participant's actions and the results in the displays [10]. In this paper we consider, for the first time, the impact of a global improvement in the display characteristics of the virtual reality system, while holding all else constant - including the content and rendering of the scenario. In particular the focus of this paper is on the impact on responses of participants to the changes caused by an upgrade to the projector system. Note that this is not concerned with realism of illumination as in other papers $[16,15]$, nor on behavioral realism $[6,1]$ but purely is concerned with the characteristics of the display.

Each participant, a supporter of English Premier League soccer team Arsenal F.C. entered a virtual bar and was approached by a virtual man wearing an Arsenal red shirt, who asks him questions

*melslater@ub.edu about the favorite soccer team of them both (Figure 1a). The virtual character is very sociable and shows through his chatter and body language that he is a big fan of Arsenal. At some point during the conversation, a second virtual man wearing a blue unbranded shirt who had been sitting nearby, stands up and starts an argument with the first one (Figure 1b), specifically denouncing support of Arsenal. Initially, the argument does not seem serious, but soon escalates and the man with the blue shirt becomes increasingly aggressive towards the virtual supporter, who, from his side, tries to defuse the situation. The experiment ends when it gets to the point of physical violence.

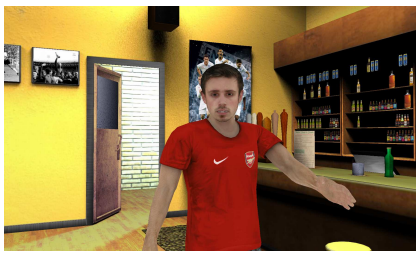

(a) The victim during the initial conversation.

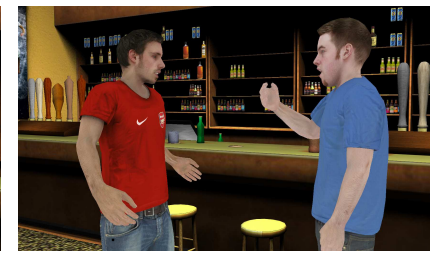

(b) Victim and aggressor during the initial stage of the confrontation.
Figure 1: Two screen shots of the scenario taken from the angle of the participant. The victim wears a red Arsenal F.C. shirt

This study uses a scenario described in our previous paper [14], where we discussed the circumstances that would make a bystander more likely to intervene in an emergency, more specifically, when they witness a violent outbreak. The earlier paper specifically studied the influence of group affiliation. Our hypothesis was that if the bystander supported the same team as the victim, then the bystander would be more likely to intervene to defuse or stop the fight, than if they were not the supporters of the same team. The results indicated that a soccer supporter is more prone to help another person wearing a shirt of the team he supports. This has also been previously observed in an experiment with real actors described in [8], but the situation was not one of a violent emergency. Perceiving the person who needs help as someone who has the same group affiliation as the bystander is a factor that is positively related to the likelihood of the bystander offering help to him. In the scenario we used for the study explained in this paper, both victim and bystander supported Arsenal.

\section{Methods}

An experiment was carried out in a Cave-like VR system. In this paper we use the term 'Cave' to refer to the generic type of system described in [5]. It has 3 walls of width $3 \mathrm{~m}$ and height $2.2 \mathrm{~m}$. The floor has a projection surface of $3 \times 3 \mathrm{~m}$. There were two different setups that we refer to as pre-upgrade and post-upgrade. By preupgrade we mean before the change of all the projectors, and by 
post-upgrade we mean after the projectors had been changed. All other aspects of the Cave system remained unchanged. In the preupgrade setup, 4 CRT projectors were used with an image resolution of $1024 \times 768$ pixels and $90 \mathrm{~Hz}$ refresh rate. The manufacturer's stated luminance for each of these projectors is 1250 Lumens. The post-upgrade setup consisted of 4 DLP projectors with a resolution of $1400 \times 1050$ pixels and $100 \mathrm{~Hz}$ refresh rate; for the floor projection the image was cropped to $1100 \times 1050$ pixels to produce an almost square projection. The manufacturer's stated luminance for each of these DLP projectors is 3150 Lumens. For both projector setups, the graphics hardware was the same (PC cluster each with NVidia Quadro FX 5600 graphics). Note that the differences in display resolution and frame rate were dictated by the specifications of the projectors in both setups. In both cases, the scenario was rendered in stereo mode and the participant was wearing Crystal Eyes shutter glasses synchronized to the projectors. An Intersense IS900 system was used to track the participant's head and adjust in real time the imagery to his perspective.

\subsection{Differences in Projector Display Characteristics}

Taken at face value, the luminance data suggest that for participants in the post-upgrade group, the illuminance was 2.5 times greater than for pre-upgrade. However there are many other factors that should be considered. Firstly, it is likely that manufacturer specifications will tend to be maximum achievable values. The luminance figures for both sets of projectors would be moderated by the actual images projected for the scenario and this was the same for both sets of projectors. Likewise, illuminance is a function of the transmission of light by the rear-projected screens (or the reflection of light by the front-projected floor screen), so also there would be the same effect for both sets of projectors. However the following aspects would not be the same:

- The DLP projectors used for the post-upgrade setup operate at almost maximum power all the time (notwithstanding small reductions to accommodate color balance across the 4 projectors). This is not the case for the CRT projectors used for the pre-upgrade setup, for which running at maximum power can burn out the phosphor of the CRTs very quickly. A rough estimate is that they operated at $75-85 \%$ of their maximum output.

- The phosphor on the surface of the CRTs of the projectors had deteriorated (they were 4 years old) and so this would have further reduced luminance.

- The luminance from projectors is also affected by the lens used - it is hard to say how much this affects either the DLP or the CRT projectors. One obvious reduction in luminance will have come from the blanking of part of the image of the DLP floor projector (post-upgrade).

The accumulated effect of these differences most likely accentuates the difference between the two sets of projectors (i.e. the ratio of luminance values is likely much greater than 2.5) since the factors stated above affect the CRT projectors more than the DLP projectors.

However, there is a further confounding aspect to this comparison, since we are really concerned with the perceived difference between the two sets of projectors. Even carefully-measured illuminance values cannot provide an accurate comparison. At the start of each trial, the participants experienced the virtual scenario for two minutes before the victim entered. During this time their eyes would have adapted, to some degree, to the lighting levels of the projection setup used.

The display resolution of the DLP projectors on the walls $(1400 \times 1050)$ yields a pixel size of $2.1 \times 2.1 \mathrm{~mm}$, while on the floor the resolution $(1100 \times 1050)$ yields a pixel size of $2.7 \times 2.8 \mathrm{~mm}$. In comparison, the resolution of the CRT projectors $(1024 \times 768)$ yields a pixel size of $2.9 \times 2.9 \mathrm{~mm}$ on the walls and $2.9 \times 3.9 \mathrm{~mm}$ on the floor.

The combination of higher illuminance and smaller pixel size in the DLP projectors produces an image that is obviously clearer and more detailed than that of the CRTs, but it is not sufficient to describe this in a quantitative way. The DLPs are more than 2.5 times brighter, and have almost double the number of pixels, than the CRT projectors; but how exactly this affects the perception of the images and the responses to a virtual environment is less quantifiable. We can speculate as to how the greater detail that can be perceived enhances our responses to the virtual world. For example being able to see facial expressions in greater detail might encourage more empathic responses to virtual characters, while a reduction in clarity or resolution might tend to break the illusion of the virtual world.

\subsection{Experiment design}

20 male participants were recruited and assigned arbitrarily to one of the two groups. All of them were strong Arsenal supporters (on the question How much do you support your team? in the recruitment questionnaire, they scored 4 or higher on a scale from 1 not at all- to 7 -very much so-). The pre-upgrade group's degree of support for Arsenal was between 4 and 7 and the post-upgrade group between 5 and 7 , with no significant difference between the groups. The age distribution of both groups was between 18 and 34 with no differences between them. The experience in VR lasted for about 7 minutes for each participant, depending on the length of the responses during the conversation that the participant had with the virtual man prior to the confrontation. The confrontation was scripted and it always had the same duration, 2 minutes and 12 seconds. Since it was scripted, nothing that participants could do would make any change in the scenario, so the confrontation would carry on regardless of their responses.

\subsection{Response variables}

The response variables observed were the number of physical interventions and the number of verbal interventions. A physical intervention was as considered any body movement that the participant made to try to catch the attention of the virtual characters, such as moving close to them, moving into their field of view, reaching out to them with the hand or placing the arms and hands between them to try to separate them. A verbal intervention was considered as the participant speaking to either one of them or to both of them. Two physical actions made at the same time were considered as just one physical intervention. A physical action made at the same time as a verbal interjection were considered as two separate interventions, one physical and one verbal. Two physical actions or two verbal interjections were considered as just one intervention if the gap between them was shorter than 3 seconds. Video coding was the primary technique to count the interventions. Participants were video recorded with their consent thus we could review their responses and know exactly what they did and said.

Immediately after the termination of the scenario participants were asked to answer some questions about the feelings and thoughts they just had during the confrontation. The questions are listed in Table 1.

\section{Results}

The response variables were counts of the number of physical (numphysical) and the number of verbal (numverbal) interventions. Box plots of the numbers of responses are shown in Fig.2. It can be seen that the distributions are highly skewed and are not Gaussian. In fact count data response variables are appropriately analysed using a log-linear Poisson regression model of the form:

$$
\log \left(\mu_{i}\right)=\beta_{0}+\sum_{j=1}^{k} \beta_{j} x_{i j}, i=1, \ldots, n
$$


Table 1: Questionnaire and Associated Variable Names. Participants had to answer, on a $1 . .7$ scale, how much each statement applied to themselves, where 1 means 'not at all' or 'at no time', and 7 means 'very much so' or 'almost all the time'

\begin{tabular}{|l|l|}
\hline Variable name & Question \\
\hline \hline Uncomfortable & $\begin{array}{l}\text { After the argument started, I was feeling un- } \\
\text { comfortable with the situation. }\end{array}$ \\
\hline Othersafety & $\begin{array}{l}\text { After the argument started I was sometimes } \\
\text { concerned for the safety of the man being } \\
\text { threatened. }\end{array}$ \\
\hline Ownsafety & $\begin{array}{l}\text { After the argument started I was sometimes } \\
\text { concerned for my own safety. }\end{array}$ \\
\hline Help & $\begin{array}{l}\text { After the argument started I looked around } \\
\text { for help. }\end{array}$ \\
\hline Otherpeople & $\begin{array}{l}\text { After the argument started I looked around } \\
\text { to check in case other people might arrive to } \\
\text { make the situation worse. }\end{array}$ \\
\hline Victimlooked & $\begin{array}{l}\text { After the argument started, the victim looked } \\
\text { at me wanting help. }\end{array}$ \\
\hline Moveaway & $\begin{array}{l}\text { After the argument started I felt I should } \\
\text { move away from those people. }\end{array}$ \\
\hline Melooking & $\begin{array}{l}\text { After the argument started, the aggressor was } \\
\text { aware of me looking at him. }\end{array}$ \\
\hline Shouldstopit & $\begin{array}{l}\text { After the argument started, I felt I should do } \\
\text { something to stop it. }\end{array}$ \\
\hline Couldstopit & $\begin{array}{l}\text { After the argument started, I felt I could do } \\
\text { something to stop it. }\end{array}$ \\
\hline Getout & $\begin{array}{l}\text { After the argument started I felt that I needed } \\
\text { to get out. }\end{array}$ \\
\hline Mindwandering & $\begin{array}{l}\text { My mind started wandering and thinking } \\
\text { about other things during the argument. }\end{array}$ \\
\hline
\end{tabular}

for $n$ observations on the response variable $y_{i}, i=1, \ldots, n$ and $k$ explanatory variables $x_{i 1}, \ldots, x_{i k}$ and each $y_{i}$ being independently Poisson distributed with the expected value $E\left(y_{i}\right)=\mu_{i}$ [9].

The independent variable here is the binary variable upgrade (pre-upgrade $=0$, post-upgrade $=1), n=20$ (10 in each upgrade condition) and the explanatory variables are the questionnaire responses. These are included in order to take account of any potential interpersonal differences between the two groups in their subjective responses to the scenario.

In order to identify the minimal set of right-hand side variables to include in the model we adopted a highly stringent stepwise approach. A stepwise regression starts by including in the fit all the variables, and then iteratively removes variables with significance $P<\alpha_{1}$, but includes variables which if added would have significance $P<\alpha_{0}$, typically with $\alpha_{0}<\alpha_{1}$. Here we chose $\alpha_{0}=0.005$ and $\alpha_{1}=0.01$. The analysis was carried out using the Stata $12^{1}$ stepwise procedure with Poisson regression. All variables, both upgrade and the questionnaire variables were of the same status in terms of inclusion or not in the final regression, so that were upgrade to be included this would be entirely the result of the stepwise procedure and was not predetermined.

The results are given in Table 2. It can be seen that the stepwise procedure includes upgrade for both response variables, and that the post-upgrade condition is significantly and positively associated with the number of interventions. Also in the case of both responses, being uncomfortable was positively associated with the number of interventions. The feeling of wanting to move away from the virtual characters was negatively associated with the number of physical interventions. However, the belief that the aggressor was aware that the participant was looking at him was associated with an increase in the number of verbal interventions. Fear by the par-

${ }^{1}$ http://stata.com/stata12/

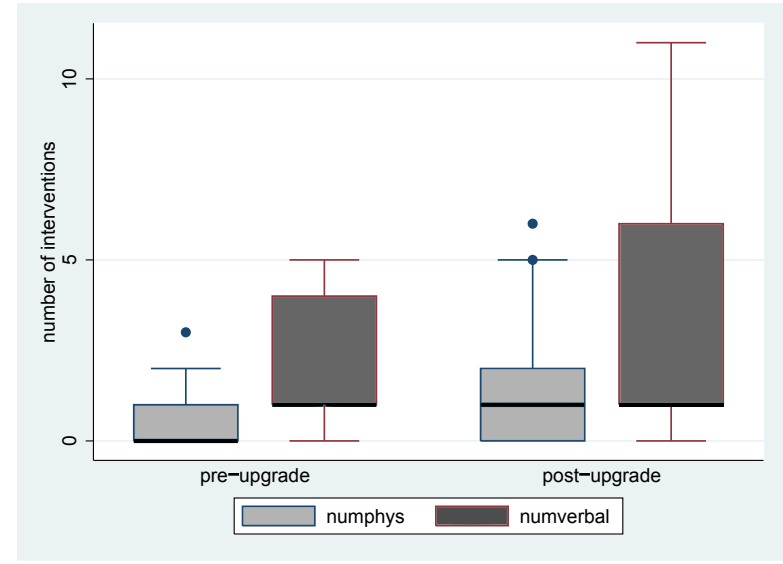

Figure 2: Box plots of the numbers of interventions by upgrade. The median is shown as the thicker line, and the box is the interquartile range. The bars extend to 1.5 the interquartile range. Points outside of the bars are shown as dots.

ticipants for their own safety was associated with a decrease in the number of interventions. In each case the greatest contributor in absolute terms was upgrade, which has the greatest absolute coefficient, which is especially the case for the number of physical interventions.

Table 2: Poisson log-linear Regressions of numphysical and numverbal on the independent variable upgrade, and the questionnaire variables from Table 1 . The final result is from a stepwise regression with bidirectional elimination with inclusion significance level of 0.005 and exclusion significance level of $0.01 . R^{2}$ is the pseudo multiple squared correlation which indicates the proportion of variance in the response explained by the model. $\chi^{2}$ is a test of goodness of fit of the model, based on the deviance of the fit, with 16 d.f. Low values of $\chi^{2}$ (high values of associated P) indicate a good fit.

\begin{tabular}{|r|r|r|l|l|l|}
\hline Variable & Coeff. $(\beta)$ & S.E. $(\beta)$ & $\mathrm{P}^{a}$ & $R^{2}$ & $\chi^{2}(16)$ \\
\hline numphysical: & & & & 0.42 & 13.0, \\
& & & & & $\mathrm{P}=0.68$ \\
upgrade & 2.44 & 0.62 & 0.000 & & \\
moveaway & -0.56 & 0.17 & 0.001 & & \\
uncomfortable & 0.61 & 0.20 & 0.003 & & \\
$\beta_{0}$ & -2.65 & 1.23 & 0.031 & & \\
\hline numverbal: & & & & 0.33 & 23.5, \\
& & & & & $\mathrm{P}=0.07$ \\
upgrade & 1.07 & 0.37 & 0.004 & & \\
uncomfortable & 0.61 & 0.15 & 0.000 & & \\
melooking & 0.97 & 0.22 & 0.000 & & \\
ownsafety & -0.70 & 0.24 & 0.004 & & \\
$\beta_{0}$ & -2.79 & 0.85 & 0.001 & & \\
\hline
\end{tabular}

${ }^{a} P=0.000$ means $P<0.0005$.

It should be noted that if we choose still more stringent values for the inclusion significance level $\alpha_{0}$ and the exclusion $\alpha_{1}$ we get very similar results. For example, consider $\alpha_{0}=0.001$ and $\alpha_{1}=0.005$. In the case of numphysical the final model fit includes upgrade $(P=$ $0.001)$ and moveaway $(P=0.001)$. In the case of numverbal the fit does not change.

One caveat that is sometimes argued (appropriately) is that for stepwise fits the final significance values are not strictly valid, since there would have been several significance tests during the stepwise 
fitting process. In fact in the case of numphysical there were 12 iterations (removal and addition of a variable) before reaching the final fit, and in the case of numverbal there were 9 such iterations. So even if we go to the extreme of adopting the Bonferroni correction of dividing the traditional 0.05 significance level by 12 and 9 respectively, the results are still well into the usual significance zone.

Table 3 shows the marginal predicted mean number of interventions, that is, after the elimination of the effects of the questionnaire variables. The results emphasize the relatively large change in the predicted number of interventions for the post-upgrade condition compared to the pre-upgrade, other things being equal.

Table 3: Predicted Marginal Number of Interventions eliminating the effect of the questionnaire variables

\begin{tabular}{|l|c|c|c|}
\hline Condition & Predicted Mean & S.E. & $95 \%$ CI \\
\hline numphysical: & & & \\
Pre-upgrade & 0.42 & 0.16 & $0.10-0.74$ \\
Post-upgrade & 4.85 & 2.06 & $0.81-8.90$ \\
\hline numverbal: & & & \\
Pre-upgrade & 1.48 & 0.34 & $0.82-2.14$ \\
Post-upgrade & 5.14 & 1.22 & $2.75-7.53$ \\
\hline
\end{tabular}

\section{Discussion}

The results show a significant difference in the number of interventions between groups. The number of both physical and verbal interventions tends to be higher in the post-upgrade version of the scenario (others things being equal). The upgrade variable is the one with a stronger effect on the number of interventions, but others extracted from the questionnaire are also important. For example, the feeling of being uncomfortable with the situation or whether the participant thought the aggressor knew he was looking at him are positively associated with the number of interventions as well. Other variables had the opposite effect. Participants who reported a strong feeling of wanting to move away or those who were concerned about their own safety intervened less.

The results of this study can be put into the context of the Place Illusion and Plausibility paradigm discussed in [13]. Place Illusion was argued to occur when sensorimotor contingencies match those of real life. In particular with head-tracked virtual reality participants can perceive the world visually through close-to-normal use of their head to change gaze direction. However, sensorimotor contingencies also depend on the display characteristics. If moving the head towards a virtual object reveals its pixelated structure this does not conform with expectations from sensorimotor contingencies in reality. Here both the luminance was greater and the pixels were smaller in the post-upgrade condition compared to the pre-upgrade. We speculate that the different behavior in the post-upgrade setting was likely due to a greater sense of being there, a component of presence, that participants would have likely felt compared to those in the pre-upgrade condition.

\section{Conclusions}

Immersive Virtual Reality has been used to place people in the situation that needs to be studied, since it has been shown that people have a tendency to respond realistically, despite the fact they know what they are in is a simulated environment. This increases the degree of ecological validity of this type of experiment compared to traditional methods such as studies based on typically abstract and definitely non-violent conflict based created in a laboratory setting. Nevertheless, special attention needs to be given to what technology is being used, since different results might be obtained from different setups and these could differ in important ways from the results obtained from real-life based experiments. The change in our projector system offered us a rare and valuable opportunity to examine the impact of such a global change in the display while holding all else constant. This has allowed an interesting insight into how such changes can apparently result in changed participant responses.

\section{ACKNOWLEDGEMENTS}

Supported by the UK EPSRC project Visual and Behavioural Fidelity of Virtual Humans with Applications to Bystander Intervention in Violent Emergencies and MS' ERC grant TRAVERSE.

\section{REFERENCES}

[1] J. N. Bailenson, K. R. Swinth, C. L. Hoyt, S. Persky, A. Dimov, and J. Blascovich. The independent and interactive effects of embodiedagent appearance and behavior on self-report, cognitive, and behavioral markers of copresence in immersive virtual environments. Presence, 14(4): pp.379-393, 2005.

[2] W. Barfield and C. Hendrix. The effect of update rate on the sense of presence within virtual environments. Virtual Reality: Research, Develpment and Application, 1(1): pp.3-16, 1995.

[3] W. Barfield, C. M. Hendrix, and K.-E. Bystrom. Effects of stereopsis and head tracking on performance using desktop virtual environment displays. Presence, 8(2): pp.237-240, 1999.

[4] D. A. Bowman, R. P. McMahan, and E. D. Ragan. Questioning naturalism in 3d user interfaces. Commun. ACM, 55(9): pp.78-88, 2012.

[5] C. Cruz-Neira, D. J. Sandin, and T. A. DeFanti. Surround-screen projection-based virtual reality: the design and implementation of the CAVE. In Proceedings of the 20th annual conference on Computer graphics and interactive techniques, SIGGRAPH '93, pp. 135-142, New York, NY, USA, 1993. ACM.

[6] M. Garau, M. Slater, V. Vinayagamoorthy, A. Brogni, A. Steed, and M. A. Sasse. The impact of avatar realism and eye gaze control on perceived quality of communication in a shared immersive virtual environment. In Proceedings of the SIGCHI conference on Human factors in computing systems, CHI '03, pp. 529-536, New York, NY, USA, 2003. ACM.

[7] W. A. IJsselsteijn, H. de Ridder, J. Freeman, S. E. Avons, and D. Bouwhuis. Effects of stereoscopic presentation, image motion, and screen size on subjective and objective corroborative measures of presence. Presence, 10(3): pp.298-311, 2001.

[8] M. Levine, A. Prosser, D. Evans, and S. Reicher. Identity and emergency intervention: How social group membership and inclusiveness of group boundaries shape helping behavior. Personality and Social Psychology Bulletin, 31(4): pp.443-453, 2005.

[9] P. McCullagh and J. A. Nelder. Generalized Linear Models. Chapman and Hall, London, UK, 1989.

[10] M. Meehan, S. Razzaque, M. Whitton, and J. Brooks, F.P. Effect of latency on presence in stressful virtual environments. In IEEE VR, 2003. Proceedings., pp. 141-148, Mar. 2003.

[11] R. Pausch, D. Proffitt, and G. Williams. Quantifying immersion in virtual reality. In Proceedings of the 24th annual conference on Computer graphics and interactive techniques, SIGGRAPH '97, pp. 1318, New York, NY, USA, 1997. ACM Press/Addison-Wesley Publishing Co.

[12] M. V. Sanchez-Vives and M. Slater. From presence to consciousness through virtual reality. Nature Reviews Neuroscience, 6(4): pp.332339, Apr. 2005.

[13] M. Slater. Place illusion and plausibility can lead to realistic behaviour in immersive virtual environments. Philos Trans $R$ Soc Lond, 364 pp.3549-3557, 2009.

[14] M. Slater, A. Rovira, R. Southern, D. Swapp, J. J. Zhang, C. Campbell, and M. Levine. Bystander responses to a violent incident in an immersive virtual environment. PLoS ONE, 8(1), Jan. 2013.

[15] I. Yu, J. Mortensen, P. Khanna, and M. Slater. Visual realism enhances realistic response in an immersive virtual environment - part 2. IEEE Computer Graphics and Applications, 32: pp.36-45, 2012.

[16] P. Zimmons and A. Panter. The influence of rendering quality on presence and task performance in a virtual environment. In IEEE VR, 2003. Proceedings., pp. 293-294, Mar. 2003. 